Article

www.mdpi.com/journal/risks

\title{
Optimal Reinsurance: A Risk Sharing Approach
}

\author{
Alejandro Balbas ${ }^{1, \star}$, Beatriz Balbas ${ }^{2}$ and Raquel Balbas ${ }^{3}$ \\ ${ }^{1}$ University Carlos III of Madrid. CL. Madrid 126. 28903 Getafe, Madrid, Spain \\ ${ }^{2}$ University of Castilla la Mancha Avda. Real Fábrica de Seda, s/n. 45600 Talavera, Toledo, Spain; \\ E-Mail: beatriz.balbas@uclm.es \\ ${ }^{3}$ University Complutense of Madrid. Department of Actuarial and Financial Economics. \\ Somosaguas-Campus. 28223 Pozuelo de Alarcón, Madrid, Spain; \\ E-Mail: raquel.balbas@ccee.ucm.es
}

* Author to whom correspondence should be addressed; E-Mail: alejandro.balbas@uc3m.es.

Received: 11 June 2013; in revised form: 21 July 2013 / Accepted: 24 July 2013 /

Published: 5 August 2013

\begin{abstract}
This paper proposes risk sharing strategies, which allow insurers to cooperate and diversify non-systemic risk. We deal with both deviation measures and coherent risk measures and provide general mathematical methods applying to optimize them all. Numerical examples are given in order to illustrate how efficiently the non-systemic risk can be diversified and how effective the presented mathematical tools may be. It is also illustrated how the existence of huge disasters may lead to wrong solutions of our optimal risk sharing problem, in the sense that the involved risk measure could ignore the existence of a non-null probability of "global ruin" after the design of the optimal risk sharing strategy. To overcome this caveat, one can use more conservative risk measures. The stability in the large of the optimal sharing plan guarantees that "the global ruin caveat" may be also addressed and solved with the presented methods.
\end{abstract}

Keywords: optimal reinsurance; general risk measure; risk sharing; systemic risk

\section{Introduction}

The optimal reinsurance problem is a classic topic in Actuarial Mathematics. A common approach is to minimize some measure of the first insurer risk after reinsurance. Seminal papers by Borch [1] and Arrow [2] used the variance as the risk measure and proved that the stop-loss reinsurance 
minimizes the retained risk if premiums are calculated with the Expected Value Premium Principle $(E V P P)$. The subsequent research followed similar ideas and tried to take into account more general risk measures and premium principles, which may give optimal contracts other than stop-loss. Recently, Gajec and Zagrodny [3] considered more general symmetric and even asymmetric risk functions, such as the absolute deviation and the truncated variance of the retained loss, under the standard deviation premium principle. Kaluszka [4] studied reinsurance contracts with many convex premium principles (exponential, semi-deviation and semi-variance, Dutch, distortion, etc.). Other well known financial risk measures, such as the Value at Risk $(V a R)$ or the Conditional Value at Risk $(C V a R$, also called $A V a R, T V a R, C T E$,Expected Shortfall, etc.), are also being considered. For example, Kaluszka [4] uses the $C V a R$ as a premium principle, and Cai and Tan [5] calculate the optimal retention for a stop-loss reinsurance by considering the $V a R$ and the $C V a R$, under the $E V P P$. Important extensions are also presented in Cai et al. [6] and Bernard and Tian [7]. Balbás et al. [8] seem to be the first authors proving that the stop-loss reinsurance is still optimal for the EVPP and the $C V a R$, though the reinsurance budget does not have to be always saturated. An interesting summary of all of these findings may be found in Centeno and Simoes [9], though there are still many open problems, and more recent papers may be found (Seng et al. [10], Cui et al. [11], Chi and Tanb [12], etc.).

It is not so usual in the literature to include the reinsurer point of view. The main reason is that the final decision is made by the ceding company. However, the reinsurer must also accept the selected strategy. A third approach considers the objectives of both companies and proposes a "reciprocal reinsurance". A very recent study may be found in Cai et al. [13], though there are more analyses involving both optimization methods and the theory of cooperative games.

This paper deals with "reciprocal reinsurance contracts" involving a general number of companies. In other words, we propose a risk sharing approach in order to diversify the risk as much as possible, so as to make the "global market risk" (or systemic risk, in this paper) as close as possible to the total sum of partial risks. Risks are measured in a very general setting, since Coherent Risk Measures (Artzner et al. [14]), Deviation Measures (Rockafellar et al. [15] and Expectation Bounded Risk Measures (Rockafellar et al. [15]) may be used.

The paper outline is as follows. Section 2 will be devoted to introducing the main notations and the framework. In Section 3, we will present an "Optimal Risk Sharing Problem" $(O R S P)$ in such a way that every insurer attempts to conserve the size of his portfolio (market share) and simultaneously reduce his risk. As said above, it may be done by means of Game Theory or Vector Optimization, and we have selected the second approach, due to the mathematical problems that the use of risk measures may cause. Thus, we propose a two stage $O R S P$, such that, first of all (stage 1), every insurer computes his ideal value (minimum risk with a non-lower market share). As usual, in Vector Optimization, the ideal values compose the Ideal Point (Nakayama et al. [16]), and a vector optimization problem (second stage) enables the insurers to look for a risk sharing plan respecting the ideal point and every market share, as well as approaching the systemic risk as much as possible.

The proposed problems are not easy to solve in practice, since it requires the minimization of general risk functions, which are barely differentiable. Standard mathematical methods do not apply, and Section 4 is devoted to extending a methodology of Balbás et al. [8,17]. We will see that Linear Programming 
linked methods will be sufficient to overcome the mathematical difficulties, despite the fact that we may deal with both discrete and continuous random variables and the risk measure is quite general.

Sections 5 and 6 present illustrative numerical examples. We only try to clarify how the developed mathematical methods allow us to solve, in practice, the proposed optimization problems in a simple manner. Simultaneously, the examples will illustrate the practical properties of the optimal risk sharing strategy and a caveat that might arise from time to time. The existence of huge disasters might lead to wrong solutions of our $O R S P$, in the sense that the involved risk measure could ignore the existence of a non-null probability of "global ruin" ( i.e., the ruin of every involved company) after the design of the optimal risk sharing strategy. To overcome this caveat, one can use more conservative risk measures. The stability in the large of the optimal sharing plan guarantees that "this global ruin caveat" may be also addressed with the presented methods.

The last section of the paper summarizes the most important conclusions.

\section{Preliminaries and Notations}

Consider the probability space, $(\Omega, \mathcal{F}, \mathbb{P})$, composed of the set of "states of the world" $\Omega$, the $\sigma$-algebra $\mathcal{F}$ and the probability measure, $\mathbb{P}$. Denote by $\mathbb{E}(y)$ the mathematical expectation of every $\mathbb{R}$-valued random variable $y$, defined on $\Omega$. Let $p \in(1, \infty)$ and denote by $L^{p}$ the Banach space of random variables, $y$, on $\Omega$, such that $\mathbb{I E}\left(|y|^{p}\right)<\infty$, endowed with the norm:

$$
\|y\|_{p}=\left(\mathbb{E}\left(|y|^{p}\right)\right)^{1 / p}
$$

for every $y \in L^{p}$. According to the Riesz Representation Theorem, $L^{q}$ is the dual space of $L^{p}$, where $q \in(1, \infty)$ is characterized by $1 / p+1 / q=1$.

Let $[0, T]$ be a time interval. From an intuitive point of view, one can interpret that $y \in L^{p}$ may represent claims at $T$ for some arbitrary insurer.

Consider $n$ insurance companies, whose final (within $[0, T]$ ) claims will be represented by the nonnegative random variables, $y_{1}, y_{2}, \ldots y_{n} \in L^{p}$, respectively. In order to simplify notations, let us assume that all of them deal with the Expected Value Premium Principle with the same loading rate, $\gamma$. Thus, the random final (at $T$ ) of the wealth of the $j^{t h}$-company will be $(1+\gamma) \mathbb{E}\left(y_{j}\right)-y_{j}, j=1,2, \ldots, n$.

In order to simplify the framework, assume that all of the insurers deal with the same risk measure:

$$
\rho: L^{p} \longrightarrow \mathbb{R}
$$

Consider the sub-gradient of $\rho$ :

$$
\Delta_{\rho}=\left\{z \in L^{q} ;-\mathbb{E}(y z) \leq \rho(y), \forall y \in L^{p}\right\} \subset L^{q} .
$$

We will assume that $\Delta_{\rho}$ is convex and $\sigma\left(L^{q}, L^{p}\right)$-compact, and:

$$
\rho(y)=\operatorname{Max}\left\{-\mathbb{E}(y z): z \in \Delta_{\rho}\right\}
$$

holds for every $y \in L^{p}$. Furthermore, we will also assume the existence of $\tilde{E}_{\rho} \geq 0$, such that the constant random variable, $z=\tilde{E}_{\rho}$, is in $\Delta_{\rho}$ and:

$$
\Delta_{\rho} \subset\left\{z \in L^{q} ; \mathbb{E}(z)=\tilde{E}_{\rho}\right\} .
$$


Summarizing, we have:

Assumption 1. The set, $\Delta_{\rho}$, given by (1) is convex and $\sigma\left(L^{q}, L^{p}\right)$-compact, (2) holds for every $y \in L^{p}, z=\tilde{E}_{\rho}$ is in $\Delta_{\rho}$ and (3) holds.

Assumption 1 is not at all restrictive, since it is satisfied by every expectation bounded risk measure (Rockafellar et al. [15]) with $\tilde{E}_{\rho}=1$ and by every deviation measure (Rockafellar et al. [15]) with $\tilde{E}_{\rho}=0$. Examples of expectation bounded risk measures are the Conditional Value at Risk $(C V a R)$ and the Weighted Conditional Value at Risk ( $W C V a R)$, amongst many others. Examples of deviation measures are, amongst others, the classical $p$-deviation:

$$
\sigma_{p}(y)=\left[\mathbb{E}\left(|\mathbb{E}(y)-y|^{p}\right)\right]^{1 / p}
$$

or the upside and downside $p$-semi-deviation:

$$
\sigma_{p}^{+}(y)=\left[\mathbb{E}\left(|\operatorname{Max}\{y-\mathbb{E}(y), 0\}|^{p}\right)\right]^{1 / p}
$$

and:

$$
\sigma_{p}^{-}(y)=\left[\mathbb{E}\left(|\operatorname{Max}\{\mathbb{E}(y)-y, 0\}|^{p}\right)\right]^{1 / p} .
$$

If $\tilde{E}_{\rho}=1$, then it is easy to see that $\rho$ is also coherent in the sense of Artzner et al. [14], if and only if:

$$
\Delta_{\rho} \subset L_{+}^{q}=\left\{z \in L^{q} ; \mathbb{P}(z \geq 0)=1\right\}
$$

Further details may be also found in Balbás et al. [8, 17].

Under the framework above, we can consider the risk of the $j^{\text {th }}$-company, given by:

$$
\rho\left((1+\gamma) \mathbb{E}\left(y_{j}\right)-y_{j}\right)
$$

$j=1,2, \ldots, n$, and the systemic risk, given by:

$$
\rho((1+\gamma) \mathbb{E}(y)-y)
$$

where:

$$
y=\sum_{j=1}^{n} y_{j} \in L^{p}
$$

denotes "global claims". Since Assumption 1 implies that $\rho$ is sub-additive, we have that:

$$
\begin{gathered}
\rho((1+\gamma) \mathbb{E}(y)-y)=\rho\left((1+\gamma) \sum_{j=1}^{n} \mathbb{E}\left(y_{j}\right)-\sum_{j=1}^{n} y_{i}\right) \\
\leq \sum_{j=1}^{n} \rho\left((1+\gamma) \mathbb{E}\left(y_{j}\right)-y_{j}\right) .
\end{gathered}
$$




\section{The Risk Sharing Problem}

Expression (6) may suggest that every company can reduce risk and simultaneously improve expected profits. Actually, companies might attempt to modify their portfolios in such a manner that (6) almost becomes an equality. This purpose may be addressed with two stages.

In the first stage, every insurer can compute his ideal risk level, i.e., the minimum risk associated with the expected wealth of his portfolio. The optimization problem for the $j^{\text {th }}$-company becomes:

$$
\left\{\begin{array}{l}
\operatorname{Min} \rho\left((1+\gamma) \mathbb{E}\left(x_{j}\right)-x_{j}\right) \\
\mathbb{E}\left(x_{j}\right) \geq \mathbb{E}\left(y_{j}\right) \\
0 \leq x_{j} \leq y
\end{array}\right.
$$

$x_{j} \in L^{p}$ being the decision variable.

Fix an ideal point,

$$
I=\left(I_{1}, I_{2}, \ldots, I_{n}\right)
$$

i.e., $I_{j}$ is not higher than the optimal value attained by (7), $j=1,2, \ldots, n$. In the second stage, all the companies, such that:

$$
I_{j}<\rho_{j}=\rho\left((1+\gamma) \mathbb{E}\left(y_{j}\right)-y_{j}\right)
$$

could collaborate, so as to improve their particular risk. Without loss of generality, we can assume that (8) holds for $j=1,2, \ldots, n$, so the insurers could look for a risk sharing plan solving the optimization problem:

$$
\begin{cases}\operatorname{Min} \sum_{j=1}^{n} w_{j} \rho\left((1+\gamma) \mathbb{E}\left(x_{j}\right)-x_{j}\right) & \\ \mathbb{E}\left(x_{j}\right) \geq \mathbb{E}\left(y_{j}\right), & j=1,2, \ldots, n \\ \sum_{j=1}^{n} x_{j} \leq y & j=1,2, \ldots, n \\ 0 \leq x_{j}, & \end{cases}
$$

$x=\left(x_{1}, x_{2}, \ldots, x_{n}\right) \in\left(L^{p}\right)^{n}$ being the decision variable and $w_{j}>0$ being a weight associated with the $j^{\text {th }}$-insurer, $j=1,2, \ldots, n$. Every company is guaranteeing that his portfolio size (or market share) will not decrease, and the weights $w=\left(w_{j}\right)_{j=1}^{n}$ must be chosen in such a manner that:

$$
I_{j}<\rho\left((1+\gamma) \mathbb{E}\left(x_{j}\right)-x_{j}\right) \leq \rho_{j},
$$

$j=1,2, \ldots, n$ must hold. Thus, every company will be approaching its ideal risk level, and the solution:

$$
x^{*}=\left(x_{1}^{*}, x_{2}^{*}, \ldots, x_{n}^{*}\right) \in\left(L^{p}\right)^{n}
$$

of (9) will be a Pareto optimum, in the sense that there is not any (9)-feasible allocation,

$$
x=\left(x_{1}, x_{2}, \ldots, x_{n}\right) \in\left(L^{p}\right)^{n}
$$

,such that:

$$
\rho\left((1+\gamma) \mathbb{E}\left(x_{j}\right)-x_{j}\right) \leq \rho\left((1+\gamma) \mathbb{E}\left(x_{j}^{*}\right)-x_{j}^{*}\right),
$$

$j=1,2, \ldots, n$, with one strict inequality at least (Nakayama et al. [16]).

Notice that the constraints of (9) lead to:

$$
\mathbb{E}\left(x_{j}\right)=\mathbb{E}\left(y_{j}\right)
$$


and:

$$
\sum_{j=1}^{n} x_{j}=y
$$

$j=1,2, \ldots, n$, so, if necessary, we can slightly modify the restrictions of (9).

\section{Mathematical Tools}

Both optimization problems, (7) and (9), involve the risk function, $\rho$, which is non-differentiable in general, and therefore, the standard mathematical methods do not apply any more. In order to overcome this caveat, several authors have proposed equivalent linear problems that apply for discrete random variables and particular interesting examples, such as the $C V a R$ or the absolute deviation (Konno et al. [18], Mansini et al. [19], etc.). Balbás et al. [8] dealt with a particular optimal reinsurance problem and gave a new linear dual problem characterizing the primal solutions and applied in a much more general setting, since the probability space, $(\Omega, \mathcal{F}, \mathbb{P})$, does not have to be discrete, and the fulfillment of Assumption 1 is the unique hypothesis about the risk measure, $\rho$. This section is devoted to pointing out how the approach of Balbás et al. [8] may be significantly extended, and both (7) and (9) have a linear dual problem characterizing their solutions. This is important from a computational point of view, since it will allow us to find, in practice, the optimal allocation, $x=\left(x_{1}, x_{2}, \ldots, x_{n}\right) \in\left(L^{p}\right)^{n}$.

With regard to Problem (7), following Balbás et al. [8], and bearing in mind (2) and (3), there is an equivalent linear problem with infinitely many constraints, namely:

$$
\left\{\begin{array}{l}
\operatorname{Min} \theta \\
\mathbb{E}\left(x_{j} z_{j}\right)-(1+\gamma) \tilde{E}_{\rho} \mathbb{E}\left(x_{j}\right) \leq \theta, \quad \forall z_{j} \in \Delta_{\rho} \\
\mathbb{E}\left(x_{j}\right) \geq \mathbb{E}\left(y_{j}\right) \\
0 \leq x_{j} \leq y
\end{array}\right.
$$

$\left(\theta, x_{j}\right) \in \mathbb{R} \times L^{p}$ being the decision variable. Indeed, bearing in mind Expression (2), one can easily prove the equivalence between (7) and (13) with quite parallel arguments to those in Balbás et al. [8]. Problem (13) shows an advantage with respect to Problem (7), because it is linear, but the drawback is provoked by the existence of infinitely many constrains (there is one constraint per every element, $z_{j} \in \Delta_{\rho}$ ). Nevertheless, the dual problem of (13) overcomes this caveat, since it remains linear and may be given as follows:

$$
\left\{\begin{array}{l}
\operatorname{Max} \mathbb{E}\left(y_{j}\right) \lambda_{j}-\mathbb{E}\left(y \nu_{j}\right) \\
z_{j}+\nu_{j} \geq(1+\gamma) \tilde{E}_{\rho}+\lambda_{j} \\
\lambda_{j} \geq 0, z_{j} \in \Delta_{\rho}, \nu_{j} \in L^{q}, \nu_{j} \geq 0
\end{array}\right.
$$

$\left(\lambda_{j}, z_{j}, \nu_{j}\right) \in \mathbb{R} \times L^{q} \times L^{q}$ being the decision variable. We can take $I_{j}$ as the optimal value of the linear dual problem above, so as to define the ideal point, $I$.

With respect to Problem (9), the equivalent linear problem with infinitely many constraints becomes:

$$
\begin{cases}\operatorname{Min} \sum_{j=1}^{n} \theta_{j} & \\ w_{j} \mathbb{E}\left(x_{j} z_{j}\right)-w_{j}(1+\gamma) \tilde{E}_{\rho} \mathbb{E}\left(x_{j}\right)-\theta_{j} \leq 0, & \forall z_{j} \in \Delta_{\rho}, j=1,2, \ldots, n \\ \mathbb{E}\left(x_{j}\right) \geq \mathbb{E}\left(y_{j}\right), & j=1,2, \ldots, n \\ \sum_{j=1}^{n} x_{j} \leq y & \\ 0 \leq x_{j}, & j=1,2, \ldots, n\end{cases}
$$


$\left(\left(\theta_{j}\right)_{j=1}^{n},\left(x_{j}\right)_{j=1}^{n}\right) \in \mathbb{R}^{n} \times\left(L^{p}\right)^{n}$ being the decision variable, while the linear dual problem is:

$$
\begin{aligned}
& \left\{\begin{array}{l}
\operatorname{Max} \sum_{j=1}^{n} \mathbb{E}\left(y_{j}\right) \lambda_{j}-\mathbb{E}(y \nu) \\
-(1+\gamma) w_{j} \tilde{E}_{\rho}+w_{j} z_{j}-\lambda_{j}+\nu \geq 0, \quad j=1,2, \ldots, n \\
\lambda_{j} \in \mathbb{R}, \lambda_{j} \geq 0, \nu \in L^{q}, \nu \geq 0, z_{j} \in \Delta_{\rho} \quad j=1,2, \ldots, n
\end{array}\right. \\
& \quad\left(\left(\lambda_{j}\right)_{j=1}^{n},\left(z_{j}\right)_{j=1}^{n}, \nu\right) \in \mathbb{R}^{n} \times L^{q} \times L^{q}
\end{aligned}
$$

being the decision variable.

We are dealing with infinite-dimensional Banach spaces, so the existence of a duality gap between (9) and (15) might hold, i.e., both problems might attain different optimal values (Luenberger, [20]). However, similar methods to those in Balbás et al. [8] enable us to prove the duality gap absence, and (15) totally characterizes the optimal allocation, $\left(x_{j}^{*}\right)_{j=1}^{n}$, solving (9). Thus, in practice, one can solve the dual problem, which is linear and, therefore, easy to solve, even if the probability space, $(\Omega, \mathcal{F}, \mathbb{P})$, is complex and (15) is an infinite-dimensional problem (Anderson and Nash, [21]), and then, one can find the optimal allocation, $\left(x_{j}^{*}\right)_{j=1}^{n}$, by applying the Karush-Kuhn-Tucker conditions below.

Suppose that $\left(x_{j}^{*}\right)_{j=1}^{n}$ is (9)-feasible and:

$$
\left(\left(\lambda_{j}^{*}\right)_{j=1}^{n},\left(z_{j}^{*}\right)_{j=1}^{n}, \nu^{*}\right)
$$

is (15)-feasible. Then, they solve (9) and (15), if and only if the following Karush-Kuhn-Tucker-like conditions:

$$
\begin{cases}\mathbb{E}\left(x_{j}^{*} z_{j}\right) \leq \mathbb{E}\left(x_{j}^{*} z_{j}^{*}\right), & \forall z_{j} \in \Delta_{\rho}, j=1,2, \ldots, n \\ \mathbb{E}\left(x_{j}^{*}\left(-(1+\gamma) w_{j} \tilde{E}_{\rho}+w_{j} z_{j}^{*}-\lambda_{j}^{*}+\nu^{*}\right)\right)=0, & j=1,2, \ldots, n \\ \mathbb{E}\left(x_{j}^{*}-y_{j}\right)=0, & j=1,2, \ldots, n \\ \sum_{j=1}^{n} x_{j}^{*}=y & \end{cases}
$$

hold.

In practice, we can consider that (16) is known, since it may be computed by solving the dual problem (15). Thus, (17) becomes a linear system of equations, leading to the optimal allocation, $\left(x_{j}^{*}\right)_{j=1}^{n}$.

\section{Numerical Experiment}

Let us deal with a simple numerical example in order to illustrate how the theorem above allows us to solve Problems (7) and (9) and obtain the optimal allocation, $\left(x_{j}^{*}\right)_{j=1}^{n}$. This numerical experiment will be used in Section 6 to point out how the optimal allocation may increase the "probability global of ruin".

Consider three companies and a set of states composed of five scenarios, $\Omega=\left\{\omega_{1}, \omega_{2}, \omega_{3}, \omega_{4}, \omega_{5}\right\}$. Suppose that the probability of all of them equals 0.2. Matrix:

$$
C_{y}=\left(\begin{array}{ccccc}
2, & 4, & 6, & 8, & 10 \\
10, & 8, & 6, & 4, & 2 \\
1, & 1, & 1, & 1, & 1000
\end{array}\right)
$$


gives the random cost for annual claims, the $j^{\text {th }}$-row being associated with the $j^{\text {th }}$-company, $j=$ 1,2,3. Suppose, finally, that $\gamma=0.05$ and that $\rho$ is the $C V a R$ with the confidence level, $60 \%$ (so $\tilde{E}_{\rho}=1$ ). Though higher levels of confidence are usually recommended, the selected one, $60 \%$, will enable us to illustrate many effects we are interested in. Furthermore, the $C V a R$ is consistent with the second order stochastic dominance (and the standard utility functions) for every level of confidence (Ogryczak and Ruszczynski, [22]).

It is easy to verify that:

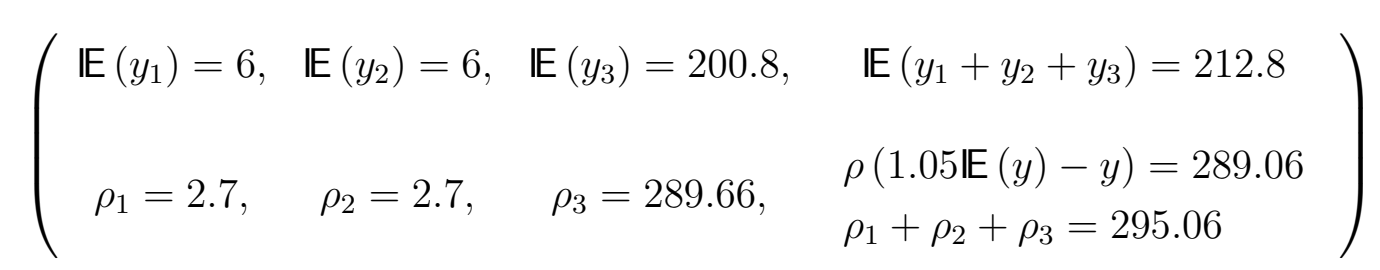

and the systemic risk, $\rho(y)=289.06$, is strictly lower than the sum of the partial risks, $\rho_{1}+\rho_{2}+\rho_{3}=$ 295.06. The three companies may attempt to remove the difference (six monetary units) by means of a risk sharing plan.

Bearing in mind that (Rockafellar et al., [15])

$$
\Delta_{\rho}=\left\{z=\left(z_{1}, \ldots, z_{5}\right) ; 0 \leq z_{i} \leq 2.5 \text { and }_{i=1}^{5} z_{i}=5\right\}
$$

Problem (14) becomes:

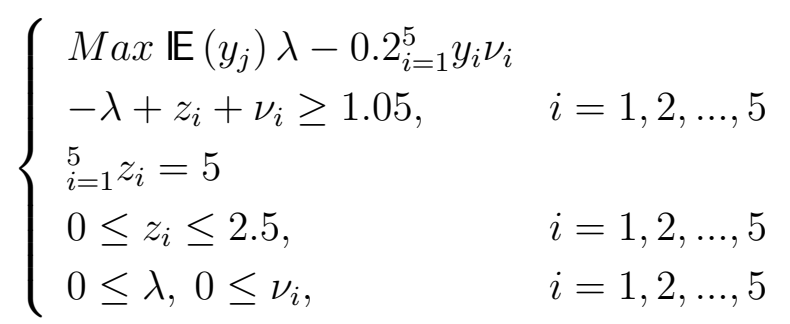

$\left(\lambda,\left(\nu_{i}\right)_{i=1}^{5},\left(z_{i}\right)_{i=1}^{5}\right) \in \mathbb{R}^{11}$ being the decision variable and $\mathbb{E}\left(y_{j}\right)$ equaling six for $j=1,2$ or 200.8 for $j=3$. By solving these simple linear problems, we can obtain the optimal value of ( 7 ) for the three involved insurers, and the ideal point becomes

$$
I=(-0.65,-0.65,271.66)
$$

As can be seen, the ideal point would significantly improve the risk level of the three companies, while their expected profit would remain the same. The first and second company could reach a negative $C V a R$, and the risk reduction could equal $\frac{2.7+0.65}{2.7}=124.07 \%$, whereas this percentage would become $\frac{289.66-271.66}{289.66} \simeq 6.21 \%$ for the third insurer. In this situation, a risk sharing plan could be interesting for them all, but recall that the ideal point above is not reachable (actually, the systemic risk, $\rho(1.05 \mathrm{IE}(y)-y)=289.06$, can never be lower than the sum of partial risks; see (6)). Besides, a significant fall of the third risk could provoke a positive increment of the rest of the ones, so the weights, 
$\left(w_{j}\right)_{j=1}^{3}$, should be selected, so as to prevent this situation. Suppose that the three companies choose $\left(w_{j}\right)_{j=1}^{3}=(10,10,1)$. Then, Problem (9) will lead to a Pareto optimum, and Problem (15) will become:

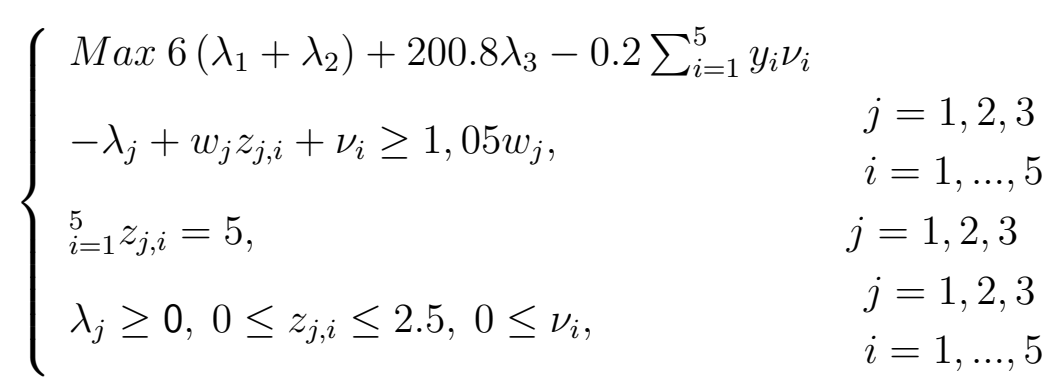

with

$$
\left(\left(\lambda_{j}\right)_{j=1}^{3},\left(\left(z_{j, i}\right)_{j=1}^{3}\right)_{i=1}^{5},\left(\nu_{i}\right)_{i=1}^{5}\right)
$$

being the decision variable and

$$
\left(y_{i}\right)_{i=1}^{5}=(13,13,13,13,1012) .
$$

Solving this problem with standard linear optimization methods and using Conditions (17), we get the optimal allocation:

$$
C_{x}=\left(\begin{array}{ccccc}
6, & 6, & 6, & 6, & 6 \\
6, & 6, & 6, & 6, & 6 \\
1, & 1, & 1, & 1, & 1000
\end{array}\right)
$$

and the summary (19) becomes:

$$
\left(\begin{array}{lll}
\mathbb{E}\left(x_{1}\right)=6, & \mathbb{E}\left(x_{2}\right)=6, \quad \mathbb{E}\left(x_{3}\right)=200.8, & \mathbb{E}\left(x_{1}+x_{2}+x_{3}\right)=212.8 \\
\rho_{1}=-0.3, \quad \rho_{2}=-0.3, & \rho_{3}=289.66, & \rho(1.05 \mathbb{E}(y)-y)=289.06 \\
\rho_{1}+\rho_{2}+\rho_{3}=289.06
\end{array}\right) .
$$

Thus, the equality:

$$
\rho(1.05 \mathrm{IE}(y)-y)=\rho_{1}+\rho_{2}+\rho_{3}
$$

shows that the three companies can totally diversify the non-systemic risk in this case. Notice that the ideal risk level is not reached by any company.

\section{Systemic Risk Reduction and Global Bankruptcy}

Consider the numerical example above, but suppose that the second stage is addressed with the alternative weights, $\left(\tilde{w}_{j}\right)_{j=1}^{3}=(1,1,1)$. Then, it is easy to repeat the process and get the new allocation:

$$
C_{\tilde{x}}=\left(\begin{array}{ccccc}
0, & 0, & 0, & 0, & 30 \\
0, & 0, & 0, & 0, & 30 \\
13, & 13, & 13, & 13, & 952
\end{array}\right) .
$$

Thus, (19) and (21) become:

$$
\left(\begin{array}{ccc}
\mathbb{E}\left(\tilde{x}_{1}\right)=6, & \mathbb{E}\left(\tilde{x}_{2}\right)=6, \quad \mathbb{E}\left(\tilde{x}_{3}\right)=200.8, & \mathbb{E}\left(\tilde{x}_{1}+\tilde{x}_{2}+\tilde{x}_{3}\right)=212.8 \\
\rho_{1}=8.7, \quad \rho_{2}=8.7, \quad \rho_{3}=271.66, & \rho(1.05 \mathbb{E}(y)-y)=289.06 \\
\rho_{1}+\rho_{2}+\rho_{3}=289.06
\end{array}\right) .
$$


Obviously, this new solution would not be accepted by the first and second insurers, since their particular risk increases from 2.7 to 8.7. As indicated in Section 4, the weights in (9) must be selected in such a manner that (10) holds. However, we could also provide other numerical examples, such that (10) would hold, and the caveat below would also apply.

Indeed, the risk sharing strategy, $C_{\tilde{x}}$, shows the existence of serious caveats that cannot be ignored when designing optimal reinsurance problems. Expression (21) implies that $C_{\tilde{x}}$ also satisfies (22), and therefore, both $C_{x}$ and $C_{\tilde{x}}$ diversify the non-systemic risk. There is only systemic risk after both reinsurance strategies. However, the major difference is caused by the state of nature, $\omega_{5} \in \Omega$. If $\omega_{5}$ comes out, then the three companies will be facing significant capital losses. In other words, if one compares Strategies $C_{y}$ and $C_{\tilde{x}}$, the probability of ruin for the three insurers is clearly higher for $C_{\tilde{x}}$, despite the fact that this risk sharing plan eliminates the non-systemic risk, while the initial one, $C_{y}$, does not.

In general, the existence of very negative catastrophes implying high capital losses generates lossdistributions very asymmetric and with very heavy tails, as well as very closely correlated risks in the insurance industry. In a risk sharing plan, it is very important to diversify the risk provoked by very severe disasters, which can only be done by dealing with very conservative risk measures, related to very risk averse decision makers. Otherwise, more aggressive risk measures will not detect this contagion effect and might indicate a global diversification of the non-systemic risk and, simultaneously, lead to risk sharing strategies, making it grow the ruin probability of the whole system.

The analysis of Balbás et al. [17] may be easily adapted to the setting of this paper. The implication is that, under weak conditions, the optimal risk sharing strategy remains stable as the risk measure becomes more and more conservative. In fact, there is a limit in the large of this strategy. Thus, the method proposed in Section 3 must be complemented with a new analysis verifying the ruin probability of the involved companies. If the result is not adequate, then the optimal strategy must be reached with a more risk averse risk measure. The stability of the optimal strategy in the large will guarantee that "the probability of global ruin" may be controlled, even when facing heavy tails and high correlations. The example in Section 5 shows that the methodology of Section 3 usually leads to successful solutions $\left(C_{x}\right.$ in (20) totally diversifies the non-systemic risk and does not make it grow any probability of ruin), but strategies, such as $C_{\tilde{x}}$, should be discarded if they were obtained.

\section{Conclusions}

The optimal reinsurance problem is a classical topic in Actuarial Mathematics. The usual viewpoint only considers the ceding company objective, though there are other approaches, taking into account the reinsurer opinion, too. This paper proposes a "reciprocal reinsurance" involving a general number of companies. The main purpose is the diversification of the non-systemic risk conserving every market share.

We have addressed the objective above by means of two stage Vector Optimization Problems. In the first step, every insurer computes his ideal risk level, and the second step provides an optimal risk sharing plan that integrates the objectives of all of the involved insurers and respects ideal points and market shares. 
The usual mathematical methods do not apply to solve the proposed optimization problems, due to the lack of differentiability, so we have provided specific mathematical tools permitting us to give explicit solutions of the presented problems. These tools apply in a very general framework, since we can deal with both discrete and continuous probability spaces, and the risk measure assumptions are quite weak.

Numerical examples have shown how the proposed tools apply in practice, as well as the properties of the reached optimal risk sharing strategy. Furthermore, the examples have shown that under some particular conditions, the selected risk sharing plan might provoke the existence of scenarios that are very negative for all of the involved companies, making it grow the "probability of global bankruptcy", with respect to this probability value, before the reinsurance contract. If so, the risk sharing plan should be modified, and a good way to do that is to choose a more conservative risk measure. The stability in the large of the optimal sharing plan guarantees that this "global ruin caveat" may be always solved.

\section{Acknowledgments}

Research partially supported by "Comunidad Autónoma de Madrid" (Spain, Grant S2009/ESP 1594) and "MEyC" (Spain, Grants ECO2009 - 14457 - C04 and ECO2012 - 39031 - C02 - 01). The usual caveat applies.

\section{References}

1. Borch, K. An attempt to determine the optimum amount of stop loss reinsurance. Transactions of the 16th International Congress of Actuaries I, 1960, 1, 597-610.

2. Arrow, K. J. Uncertainty and the welfare of medical care. Am. Econ. Rev. 1963, 53, 941-973.

3. Gajec, L.; Zagrodny, D. Optimal reinsurance under general risk measures. Insur. Math. Econ. 2004, 34, 227-240.

4. Kaluszka, M. Optimal reinsurance under convex principles of premium calculation. Insur. Math. Econ. 2005, 36, 375-398.

5. Cai, J.; Tan, K.S.. Optimal retention for a stop loss reinsurance under the $V a R$ and $C T E$ risk measures. ASTIN Bull. 2007, 37, 93-112.

6. Cai, J.; Tan, K.S.; Weng, C.; Zhang, Y. Optimal reinsurance under $V a R$ and $C T E$ risk measures. Insur. Math. Econ. 2008, 43, 185-196.

7. Bernard, C.; Tian, W. Optimal reinsurance arrangements under tail risk measures. J. Risk Insur. 2009, 76, 709-725.

8. Balbás, A.; Balbás, B.; Heras, A. Optimal reinsurance with general risk measures. Insur. Math. Econ. 2009, 44, 374-384.

9. Centeno, M.L.; Simoes, O. Optimal reinsurance. RACSAM 2009, 103, 387-405.

10. Seng, K.T.; Wenga, C.; Zhang, Y. Optimality of general reinsurance contracts under CTE risk measure. Insur. Math. Econ. 2011, 49, 175-187.

11. Cui, W.; Yang, J.; Wu, L. Optimal reinsurance minimizing the distortion risk measure under general reinsurance premium principles. Insur. Math. Econ. 2013, 53, 74-85.

12. Chi, Y.; Tanb, K.S. Optimal reinsurance with general premium principles. Insur. Math. Econ. 2013, 52, 180-189. 
13. Cai, J.; Fang, Y.; Li, Z.; Willmot, G.E. Optimal reciprocal reinsurance treaties under the joint survival probability and the joint profitable probability. J. Risk Insur. 2012, 80, 145-168.

14. Artzner, P.; Delbaen, F.; Eber, J.M.; Heath, D. Coherent measures of risk. Math. Finance 1999, 9, 203-228.

15. Rockafellar, R.T.; Uryasev, S.; Zabarankin, M. Generalized deviations in risk analysis. Financ. Stoch. 2006, 10, 51-74.

16. Nakayama H.; Sawaragi, Y.; Tanino, T. Theory of Multiobjective Optimization; Academic Press: Waltham, MA, USA, 1985.

17. Balbás, A.; Balbás, B.; Heras, A. Stable solutions for optimal reinsurance problems involving risk measures. Eur. J. Oper. Res. 2011, 214, 796-804.

18. Konno, H.; Akishino, K.; Yamamoto, R. Optimization of a long-short portfolio under non-convex transaction costs. Comput. Optim. Appl. 2005, 32, 115-132.

19. Mansini, R.; Ogryczak, W.; Speranza, M.G. Conditional value at risk and related linear programming models for portfolio optimization. Ann. Oper. Res. 2007, 152, 227-256.

20. Luenberger, D.G. Optimization by Vector Spaces Methods; John Wiley \& Sons: Hoboken, NJ, USA, 1969.

21. Anderson, E.J.; Nash, P. Linear Programming in Infinite-Dimensional Spaces; John Wiley \& Sons: Hoboken, NJ, USA, 1987.

22. Ogryczak, W.; Ruszczynski, A. Dual stochastic dominance and related mean risk models. SIAM J. Optimiz. 2002, 13, 60-78.

(C) 2013 by the authors; licensee MDPI, Basel, Switzerland. This article is an open access article distributed under the terms and conditions of the Creative Commons Attribution license (http://creativecommons.org/licenses/by/3.0/). 